\title{
Near-infrared survey of high mass X-ray binary candidates
}

\author{
J. M. Torrejón ${ }^{1,2}$, I. Negueruela ${ }^{1}$, D. M. Smith ${ }^{3}$, and T. E. Harrison ${ }^{4}$
}

\author{
1 Departamento de Física, Ingeniería de Sistemas y Teoría de la Señal, Universidad de Alicante, 03080 Alicante, Spain \\ e-mail: jmt@ua.es \\ 2 Massachusetts Institute of Technology, Kavli Institute for Astrophysics and Space Research, Cambridge MA 02139, UK \\ 3 Physics Department and Santa Cruz Institute for Particle Physics, University of California, Santa Cruz, 1156 High St., Santa Cruz, \\ CA 95064, USA \\ 4 Astronomy Department, New Mexico State University, Box 30001/Department 4500, Las Cruces, NM 88003, USA
}

Received 2 June 2009 / Accepted 18 October 2009

\begin{abstract}
Context. The INTEGRAL satellite is detecting a large population of new X-ray sources that were missed by previous missions because of high obscuration and, in some cases, very short duty cycles. The nature of these sources must be addressed by characterizing their optical and/or infrared counterparts.

Aims. We investigate the nature of the optical counterparts to five of these newly discovered X-ray sources.

Methods. We combine infrared spectra in the $I, J, H$, and $K$ bands with $J H K$ photometry to characterize the spectral type, luminosity class, and distance to the infrared counterparts to these systems. For IGR J19140+0951, we present spectroscopy from the red to the $K$ band and new red and infrared photometry. For SAX J18186-1703 and IGR J18483-0311, we present the first intermediateresolution spectroscopy to be published. Finally, for IGR J18027-2016, we present new $I$ and $K$ band spectra.

Results. We find that four systems harbour early-type B supergiants. All of them are heavily obscured, with $E(B-V)$ ranging between 3 and 5, implying visual extinctions of $\sim 9$ to 15 mag. We refine the published classifications of IGR J18027-2016 and IGR J19140+0951 by constraining their luminosity class. In the first case, we confirm the supergiant nature but exclude a class III. In the second case, we propose a slightly higher luminosity class (Ia instead of Iab) and provide an improved value of the distance based on new optical photometry. Two other systems, SAX J18186-1703 and IGR J18483-0311 are classified as supergiant fast X-ray transients (SFXTs). XTE J1901+014, on the other hand, contains no bright infrared source in its error circle.

Conclusions. Owing to their infrared and X-ray characteristics, IGR J18027-2016 and IGR J19140+0951, emerge as supergiant X-ray binaries with X-ray luminosities of the order of $L_{\mathrm{X}} \sim[1-2] \times 10^{36} \mathrm{erg} \mathrm{s}^{-1}$, while SAX J1818.6-1703 and IGR J18483-0311, are found to be SFXTs at 2 and $3 \mathrm{kpc}$, respectively. Finally, XTE J1901+014 emerges as a puzzling source: its X-ray behaviour is strongly reminiscent of SFXTs, but a supergiant nature is firmly excluded for the counterpart. We discuss several alternative scenarios to explain its behaviour.
\end{abstract}

Key words. binaries: close - supergiants - infrared: stars - X-rays: binaries

\section{Introduction}

The INTEGRAL satellite has broadened our knowledge of X-ray binaries, by discovering a significant population of new X-ray sources. The third IBIS/ISGRI cataloge (Bird et al. 2007) contains more than 420 sources of which 167 are new. The online cataloge of INTEGRAL sources $^{1}$ lists 245 sources that have been discovered or rediscovered by INTEGRAL. Of these, $61(\sim 25 \%)$ remain unidentified and $81(\sim 33 \%)$ are extragalactic objects (e.g., AGNs, Seyferts, QSOs). The reminder are mainly X-ray binaries. Fifty one of these sources $(\sim 21 \%)$ were found to be high mass X-ray binary candidates (HMXB), missed by previous missions because of their high obscuration, their very short X-ray cycles, or a combination of both. Approximately half of them have been independently confirmed so far. Amongst these new 51 HMXB systems, there are a few Be/X-ray binaries $(\mathrm{BeXB})$, supergiant $\mathrm{X}$-ray binaries (SGXB) and the newly established class of supergiant fast X-ray transients (SFXT; e.g., Smith et al. 2006; Negueruela et al. 2006a; Sguera et al. 2006). This last class comprises 16 candidates of which nearly half have been confirmed. Before we can conclusively classify every new

\footnotetext{
1 http://isdcul3. unige. ch/ rodrigue/html/igrsources . html
}

source and understand its X-ray behavior, we must first establish the nature of the counterpart.

Since the majority of these sources are located in the Galactic plane, and concentrated towards the Galactic center region, they are affected by high extinction $\left(A_{V} \lesssim 15 \mathrm{mag}\right)$. Thus, their detection in the optical is often very difficult, and other strategies must be adopted. The detection and study of counterparts in the infrared, however, is possible with $4 \mathrm{~m}$ class telescopes. An important caveat, though, is that the spectral classification of hot stars using infrared spectra is far more uncertain than using the well established MK standard 3950-4750 ̊ wavelength region. For example, a spectral classification based on a $K$ band spectrum cannot be completed without fundamental ambiguities because of the lack of adequate spectral features in that range (Hanson et al. 1996). This problem can be, at least partially, circumvented by combining data for several spectral bands. However, this is often impossible to achieve within a single observing campaign.

Following the discovery of X-ray binaries by INTEGRAL, intense observing campaigns have been undertaken (Chaty et al. 2008; Masetti et al. 2008; Nespoli et al. 2008; Negueruela et al. 2009, amongst others). As these discoveries proceed, it is fundamental to refine and establish the nature of these new sources. In particular, a very important parameter is the distance to the 
Table 1. $J H K^{\prime}$ infrared photometry for the sources included in our survey.

\begin{tabular}{lccclccc}
\hline \hline Source & 2MASS & RA & Dec & Ref. & $J(1.27 \mu \mathrm{m})$ & $H(1.63 \mu \mathrm{m})$ & $K^{\prime}(2.12 \mu \mathrm{m})$ \\
\hline IGR J18027-2016 & 2MASS J18024194-2017172 & 180241.94 & -201717.3 & $\mathrm{C}$ & $12.79 \pm 0.06$ & $11.96 \pm 0.08$ & $11.48 \pm 0.05$ \\
SAX J18186-1703 & 2MASS J18183790-1702479 & 181837.90 & -170247.9 & $\mathrm{Z} 1$ & $10.08 \pm 0.06$ & $8.94 \pm 0.08$ & $7.79 \pm 0.05$ \\
IGR J18483-0311 & 2MASS J18481720-0310168 & 184817.20 & -031016.8 & $\mathrm{C}$ & $10.84 \pm 0.03$ & $9.38 \pm 0.02$ & $8.47 \pm 0.02$ \\
IGR J19140+0951 & 2MASS J19140422+0952577 & 191404.23 & +095257.7 & $\mathrm{Z} 2$ & $11.33 \pm 0.06$ & $9.71 \pm 0.08$ & $8.83 \pm 0.05$ \\
\hline
\end{tabular}

Notes. ${ }^{(a)}$ Photometry from Rahoui \& Chaty (2008). For this source, the filters are centered on $1.25,1.65$ and $2.20 \mu$ m respectively.

References. C: Chaty et al. (2008); Z1: in’t Zand et al. (2006a); Z2: in’t Zand et al. (2006b).

system which, in turn, infers the X-ray luminosity, the main observable used to constrain the theoretical models. Most of the work done, however, has been based on $H$ and/or $K$ band spectra to avoid the problems caused by the high extinction. Classification based on a single infrared spectrum (in many cases of an intermediate or low spectral resolution) allows only approximations. For example, the difference in $M_{V}$ between a $\mathrm{B} 1 \mathrm{Ib}$ and a B1 Ia supergiant (which are difficult or even impossible to distinguish based on an intermediate resolution $H$ or $K$ spectrum) results in an uncertainty of an order of magnitude error in $L_{X}$ (Negueruela et al. 2009). In this paper, we combine new spectroscopy in several bands with new $J H K$ photometry (also $R I$ for one source) to refine the spectral classification of a sample of these sources.

\section{The observations}

\subsection{Photometry}

$J H K^{\prime}$ band photometry data were obtained with the $3.5 \mathrm{~m}$ Telescopio Nazionale Galileo (TNG) at the island of La Palma (Canary Islands, Spain) during the nights of 2006 July 7 and 2007 May 1. In both runs, the configuration was the same. The instrument used was the Near Infrared Camera and Spectrograph (NICS) in its Large Field configuration, equipped with a Rockwell $1024 \times 1024 \mathrm{HgCdTe}$ Hawaii array detector, which provides a spatial scale of $0.25 /$ pixel and covers a field of view of $4.2 \times 4.2$. During the night of 2006 July 7, the seeing was 0.7 in the $J$ band and very stable throughout the entire night, while on 2007 May 1, the seeing was 0.'8.

Each observation consisted of a mosaic of five images constructed from a dithering pattern that maintained the frame always on-source, using an automatic script available at the telescope. From this mosaic, flat-field correction, bad pixel map, sky subtraction, and image coaddition where performed with the SNAP software ${ }^{2}$. The photometry was subsequently performed using Starlink GAIA on the final coadded frame.

The Arnica standard star fields AS33 and AS27 (Hunt et al. 1998) were observed throughout the night at a range of airmasses. Transformation equations were applied to the program star giving the magnitudes listed in Table 1.

Comparison with previously published values show some discrepancies that can be readily explained. In particular, for IGR J19140+0951, comparison with 2MASS magnitudes shows a discrepancy. This is because of the close proximity of the bright star 2MASS J19149417+0952538, located very close to the south-east, which contaminates the 2MASS photometry (cf. in't Zand et al. 2006b). Any photometry performed using data from a $3.5 \mathrm{~m}$ telescope in good seeing conditions must certainly be contaminated. However, careful attention must be paid

\footnotetext{
2 http://www.tng.iac.es/news/2002/09/10/snap/index. html
}

here to the extraction to minimize this effect, making the magnitudes listed above rather more reliable than those of the 2MASS catalogue.

For IGR J19140+0951, RI band photometry was obtained with the Andalucia Faint Object Spectrograph and Camera (ALFOSC) on the $2.6 \mathrm{~m}$ Nordic Optical Telescope (NOT) in La Palma, on the night of 2006 June 18. The instrument was equipped with the thinned $2048 \times 2048$ pixel E2V CCD, covering a field of view of $6.4 \times 6.4$ with a spatial scale 0.19 arcsec/pixel. Standard Bessel $R$ and interference $i$ filters were used to observe both the target and the standard star fields. We obtain $R=20.77 \pm 0.05$ and $I=17.30 \pm 0.03$.

\subsection{Spectroscopy}

Infrared spectroscopy of several sources was performed with NICS. Medium-resolution infrared spectroscopy in the $K$ band was also obtained on 2006 July 7 . The instrument was equipped with the $K_{\mathrm{b}}$ grism, which covers the 1.95-2.34 $\mu \mathrm{m}$ spectral region, and provides a resolution of $4.3 \AA /$ pixel. A slit of $1^{\prime \prime}$ was chosen, providing a resolving power of $R=1250$.

To estimate the sky background level, we observed the target stars, integrating on four positions along the slit. We used the $A B B A$ script that automatically moves the telescope, nodding the source along the slit, thereby producing a set of four images with typical exposure times ranging from 100 to $300 \mathrm{~s}$ for the programme stars and minimising the sky variability throughout each observation. The four images were median averaged and this median, subtracted from each individual image of the set. This method, together with the use of the 1 " slit, also minimizes any possible nebular contamination. These sky-subtracted images were flat-fielded and the resulting spectra averaged. The wavelength calibration was performed using the Ar lamps available at the telescope. Analysis was subsequently performed with Starlink FIGARO and DIPSO.

A number of $\mathrm{A} 0 \mathrm{~V}$ and $\mathrm{G} 2 \mathrm{~V}$ stars were selected from the list available at the telescope and observed throughout the night at airmasses and positions as close as possible to the target fields.

For the telluric subtraction, two methods were used. The first one, described in Hanson et al. (1996), assumes that spectra of A0 V stars are featureless in the $K$ band except for the $\operatorname{Br} \gamma$ line. A spectrum from the Sun was retrieved from the ESO archives. This spectrum was rebinned to match the sampling of our target spectra, wavelength shifted for the different radial velocities, and, finally, the lines rotationally broadened to match the profiles of our G2 V standard stars. The spectrum of our G2 V stars was then divided by this corrected spectrum of the Sun. The resulting spectrum was subsequently used to correct the $\operatorname{Br} \gamma$ zone in the spectrum of the $\mathrm{A} 0 \mathrm{~V}$ standard star, isolating the true $\mathrm{Br} \gamma$ profile of the early-type star. We then, fitted this line only and removed it from the A0 V spectrum, leaving a pure telluric spectrum which was subsequently applied to the program stars. 
Table 2. Equivalent widths ( $\AA$ ) of the main diagnostic lines in the $J H K$ bands observed in our sample.

\begin{tabular}{|c|c|c|c|c|c|c|c|c|}
\hline Source & He I $2.058 \mu \mathrm{m}$ & He I 2.1126 & N III 2.1155 & $\operatorname{Br} \gamma 2.1661$ & He I 1.083 & $\mathrm{~Pa} \beta 1.2818$ & Br 111.6814 & He I 1.7004 \\
\hline IGR J18027-2016 & $\begin{array}{c}4.1(\mathrm{a}+\mathrm{e}) \\
-4.5(\mathrm{e})\end{array}$ & 2.6 & -0.2 & $\begin{array}{l}1.1(\mathrm{a}+\mathrm{e}) \\
-4.1(\mathrm{e})\end{array}$ & $\ldots$ & $\ldots$ & $\ldots$ & $\ldots$ \\
\hline SAX J18186-1703 & $\begin{array}{c}8.6(a+e) \\
-6.5(e)\end{array}$ & 2.0 & $\ldots$ & 5.1 & $\ldots$ & $\ldots$ & $\ldots$ & $\ldots$ \\
\hline IGR J19140+0951 ${ }^{\dagger}$ & $\begin{array}{c}-1.9(\mathrm{a}+\mathrm{e}) \\
-5.7(\mathrm{e})\end{array}$ & 2.2 & $\ldots$ & $\begin{array}{c}2.0(\mathrm{a}+\mathrm{e}) \\
-1.0(\mathrm{e})\end{array}$ & $\ldots$ & $\ldots$ & $\ldots$ & $\ldots$ \\
\hline IGR J19140+0951 & $\begin{array}{c}-1.1(\mathrm{a}+\mathrm{e}) \\
-1.3(\mathrm{e})\end{array}$ & 2.2 & -0.4 & $\begin{array}{l}1.2(\mathrm{a}+\mathrm{e}) \\
-2.6(\mathrm{e})\end{array}$ & -7.1 & $\begin{array}{c}1.8(\mathrm{a}+\mathrm{e}) \\
-5.3(\mathrm{e})\end{array}$ & 2.3 & 2.1 \\
\hline
\end{tabular}

Notes. $(a+e)$ : absorption + emission; (e): emission only.

(†) T NG data; () S PEX-IRTF data.

The second method is described in Maiolino et al. (1996), which uses the G2 V star spectrum divided by the modified solar spectrum directly to correct the whole spectrum of the program star. At the resolution of our spectra, these two methods provide almost indistinguishable results. Where appropriate, we averaged the final spectra, of each source, obtained by both methods. The resulting spectra were normalised to unity to allow comparison with standard atlases.

In addition, infrared spectroscopy of the counterpart to IGR J19140+0951 was obtained using SPEX on the Infrared Telescope Facility (IRTF) on Mauna Kea on 2005 September 3. The $J, H$, and $K$ band spectra presented in Fig. 4 were constructed from medians of six $10 \mathrm{~s}$ exposures. The observing and data reduction procedure are fully described in Harrison et al. (2004). SPEX was used in single-order mode with a 0.3 slit, giving a dispersion of $5.51 \AA$ /pixel. Unfortunately, the conditions at the IRTF were not photometric, despite the seeing being excellent.

We employed a script with which data at six separate positions along the slit were obtained. To remove the sky background and dark current from each SPEX exposure, we subtracted the median of the other five exposures obtained in an observing sequence. This process resulted in six background-subtracted exposures from which the spectra were extracted using the normal IRAF methods. The spectra were wavelength calibrated by the extraction of an Ar arc spectrum at the position (aperture) of each spectrum. We observed the G2 V star HD 150698 (in an identical fashion) to correct for telluric features as described above.

Finally, I-band spectra of IGR J18027-2016 and IGR J18483-0311 were obtained on 2008 May 16 using the 4.2-m William Herschel Telescope (WHT), in La Palma (Spain) equipped with the ISIS double-beam spectrograph, during a service run. We used the red arm fitted with the R316R grating and the RED+ CCD, a configuration that results in a nominal dispersion of $0.85 \AA$ /pixel (the resolution element is approximately 3 pixels). We also observed the counterpart to IGR J19140+0951 on 2008 May 18 using the same instrument, but this time fitted with the R158R grating, which results in a nominal dispersion of $1.8 \AA$ À/pixel.

\section{Results}

For the line identification and spectral classification, we used the following atlases: Andrillat et al. (1995) for the $I$ band; Wallace et al. (2000) for the $J$ band; Blum et al. (1997),
Meyer et al. (1998) and Hanson et al. (1998) for the $H$ band; Hanson et al. (1996) and Hanson et al. (2005) for the $K$ band.

As has been pointed out, the classification of massive stars using the 2 micron spectral range is difficult because of the lack of spectral features in this domain. In the case of HMXB, the situation is still worse because the spectra of the infrared counterparts might be altered by the presence of the compact object in the system. Little progress has been made in obtaining nearinfrared observations of HMXBs (see, for example, Clark et al. 1999; Fig. 7, for some BeXs). In the pioneering work by Hanson et al. (1996) a number of HMXB are included (cf. Fig. 29 in that reference). In almost all of them, the relevant diagnostic lines appear in emission, in both BeXs and SGXBs. However, isolated supergiants tend to exhibit these lines in absorption (Hanson et al. 1996). No HMXBs are included in the higher S/N survey of Hanson et al. (2005). Notwithstanding, this last work has been used to classify the infrared counterparts of some HMXBs (Mason et al. 2009). In the abscence of a comprehensive scheme for classifying HMXB counterparts on the basis of near-infrared spectra, we use the previously cited available atlases. We estimate that the uncertainty caused by the above-mentioned effects alone could be equivalent to half a spectral subtype.

In Table 2, we provide the equivalent widths of the main diagnostic lines found in our spectra. Typical errors are always less than $1 \AA$. Numbers beside chemical species are the laboratory wavelengths in $\mu \mathrm{m}$. I band equivalent widths have not been measured due to the line blending. Due caution must be exercised, however, in using them only for spectral classification purposes, as stated by Hanson et al. (2005, ;see their Sect. 5.2) because only the comparison between different classes appears to be meaningful.

In order to compute their distance and $L_{X}$, we will use the infrared distance modulus computed from the following equation:

$K_{0}-M_{K}=5 \log d-5$,

where $K_{0}=K-A_{K}$ are the infrared magnitudes corrected for extinction, and the $K$ magnitudes are taken from Table 1 . We compute the $M_{K}$ band absolute magnitudes from the $(V-K)_{0}$ colors given by Ducati et al. (2001), taking into account the $M_{V}$ values from Schmidt-Kaler (1982). To compute the totalto-selective absorption $A$, we first compute the corresponding IR color excess $E(J-K)=(J-K)-(J-K)_{0}$, where the intrinsic IR colors are taken from Ducati et al. (2001) for the appropriate spectral type. These excesses are converted to $E(B-V)$ by means of the following relation $E(J-K)=0.50 E(B-V)$. 
A\&A 510, A61 (2010)

Table 3. Derived parameters for stars in our sample, calculated using the observed data.

\begin{tabular}{llccccccr}
\hline \hline Source & Spectral type & Class & $E(B-V)$ & $E(J-K)$ & $M_{V}$ & $M_{K}$ & $d(\mathrm{kpc})$ & $L_{\mathrm{X}}(\mathrm{erg} / \mathrm{s})^{a}$ \\
\hline IGR J18027-2016 & B1 Ib & SGXB & $3.04 \pm 0.02$ & 1.54 & -5.8 & -5.1 & $12.4 \pm 0.1$ & $(1.65 \pm 0.03) \times 10^{36}$ \\
SAX J18186-1703 & B0.5 Iab & SFXT & $5.08 \pm 0.05$ & 2.54 & -6.4 & -5.65 & $2.1 \pm 0.1$ & $(8 \pm 0.4) \times 10^{35}$ \\
IGR J18483-0311 & B0-1 Iab & SFXT & $5.22 \pm 0.02$ & 2.61 & -6.4 & -5.66 & $2.83 \pm 0.05$ & $(1.9 \pm 0.06) \times 10^{36}$ \\
IGR J19140+0951 & B0.5 Iab-a & SGXB & $5.5 \pm 0.1$ & 2.75 & $-6.65 \pm 0.03$ & $-5.90 \pm 0.25$ & $3.6 \pm 0.04$ & $(1.6 \pm 0.3) \times 10^{36}$ \\
XTE 1901+014 & G5 III - A3 V & LMXB? & $\sim 3.7$ & 1.8 & & & $2-7$ & $1_{-0.8}^{+1} \times 10^{38}$ \\
\hline
\end{tabular}

Notes. ${ }^{(a)} \mathrm{O}$ bserved peak luminositites for the energy ranges (2-10) keV, (22-55) keV, (20-100) keV, (2-20) keV and (3-12) keV respectively.
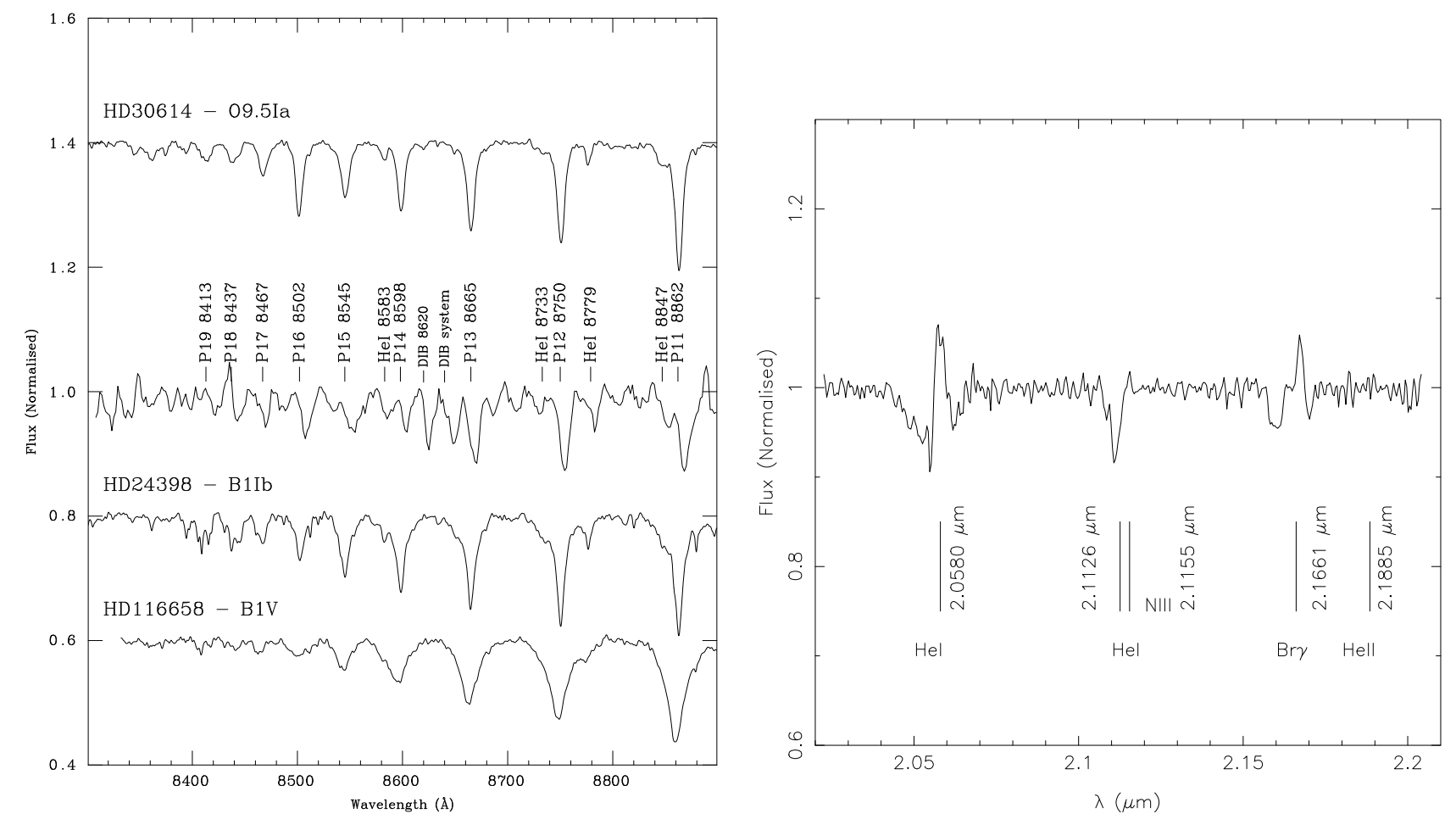

Fig. 1. Left: WHT I-band spectrum of IGR J18027-2016 normalised to flux unity bracketted between comparison standard stars. Right: TNG $K$-band spectrum normalised to flux unity.

Finally $A_{K}=0.36 E(B-V)$ (Fitzpatrick 1999), assuming the standard extinction law $\left(R_{V}=3.1\right)$. We compute the distance for the two extremes of the range in spectral type compatible with our observations, namely, the cold-faint and hot-luminous ends. The range of possible distances deduced in this way and the corresponding X-ray luminosities are given in Table 3. The quoted errors refer only to the dispersion in these estimated values. The final uncertainties in the distance are dominated by the intrinsic dispersion of the calibration of the order of $0.5 \mathrm{mag}$.

\subsection{IGR J18027-2016}

This source was discovered by Revnivtsev et al. (2004) using INTEGRAL. The source has a very high hydrogen column $\left(N_{\mathrm{H}}=(6.8 \pm 1) \times 10^{22} \mathrm{~cm}^{-2}\right)$. It has been identified with SAX J1802.7-2017, which was discovered serendipitously by Augello et al. (2003) with BeppoSAX. Using XMM-Newton, Walter et al. (2006) localised the source position at $\alpha=$ $18 \mathrm{~h} 02 \mathrm{~m} 42.0 \mathrm{~s}, \delta=-20^{\circ} 17^{\prime} 18^{\prime \prime}$ with a $4^{\prime \prime}$ positional accuracy. Its $2-10 \mathrm{keV}$ unabsorbed flux during the primary pulse is $8.9 \times 10^{-11} \mathrm{erg} \mathrm{s}^{-1} \mathrm{~cm}^{-2}$. It is an eclipsing X-ray pulsar $\left(P_{\text {spin }}=\right.$ $139.612 \mathrm{~s}$ ), which orbits around the donor star each $4.6 \mathrm{~d}$ (Hill et al. 2005).
Based on the orbital solution, Hill et al. (2005) proposed that the mass donor should be an early-type supergiant with most likely parameters $M_{*} \sim 21 M_{\odot}$ and $R_{*} \sim$ $19 R_{*}$. Masetti et al. (2008) identified the counterpart as 2MASS J18024194-2017172 and obtained a low-resolution optical spectrum that showed it to be a very reddened early-type star. Using the mass and radius deduced by Hill et al. (2005), they argued that the counterpart should be a B-type giant. Chaty et al. (2008) confirmed the identity of the counterpart. Their infrared spectrum seems typical of an early-B supergiant, displaying prominent Brackett lines in the $H$ band, even though these authors claimed the detection of He I and He II lines in the optical spectrum. They favor a B type supergiant based on a $T_{\text {eff }} \sim 20800 \mathrm{~K}$ deduced from a fit to the SED. Based on the $R_{*} / D$ value of the normalization, and assuming a stellar radius $R_{*}=20 R_{\odot}$, they estimated a distance of $\sim 12 \mathrm{kpc}$. Chaty et al. (2008) estimated that $A_{V} \sim 8.8$ mag, while Masetti et al. (2008) estimated $\sim 8.3 \mathrm{mag}$. In the following, we use our TNG $K$-band and WHT $I$-band spectra (Fig. 1) to refine the classification of the counterpart and establish the nature of the system.

$K$ BAND SPECTRUM. The presence of He I lines and the absence of He II (which is seen up to O9) points towards a $\mathrm{B}$ type star. The $\mathrm{Br} \gamma$ line is in emission. This line seems to be 
blended with the blueward absorption of the He I $2.161 \mu \mathrm{m}$ line. This morphology is more pronounced in high luminosity B stars (Hanson et al. 2005, Fig. 12). We also observe He I $2.058 \mu \mathrm{m}$ in emission, with a possible P-Cygni profile, a feature also noted by Chaty et al. (2008) on their lower resolution spectrum. In the atlas of Hanson et al. (2005), no luminosity class III star shows $\mathrm{He}$ I $2.058 \mu \mathrm{m}$ in emission. In contrast, this line is in emission in B supergiants and Be stars. However, He I $2.113 \mu \mathrm{m}$ is not seen in Be stars, while it is very prominent in our object, a feature of B supergiants (see Figs. 13 to 16 in Hanson et al. 2005). We therefore conclude that the counterpart is an early B supergiant star.

$I$ BAND SPECTRUM. As seen in Fig. 1, our $I$-band spectrum has a low signal-to-noise ratio (SNR). However, it is easy to see the narrow well-defined Paschen lines, typical of a highluminosity object. Moreover, Paschen lines are visible up to $\mathrm{Pa} 19$, in spite of the moderate SNR and resolution, clearly identifying the star as a supergiant (compare to the B1 V star in the same figure). The prominent $\mathrm{He}$ I lines are typical of supergiants with spectral types close to B2 (compare to the earlier supergiants in the same figure). Also, as can be seen in the spectra of the O9.5 Ia star, for stars B0.5 and earlier, the C III $8502 \AA$ is blended with $\mathrm{Pa} 16$, making it stronger than the neighbouring $\mathrm{Pa} 15$ and $\mathrm{Pa}$ 17. This is not the case in our object, suggesting it is later than B0.5. On the other hand, the red spectrum of IGR J18027-2017 does not display O I $7774 \AA$ (which is not seen either in the spectrum of Chaty et al. (2008)). This would indicate a type earlier than B1.5 if it is a luminous supergiant or earlier than B2 if it is less luminous.

Taking all the data together, we propose a B1 Iab-B1 Ib supergiant counterpart. Discriminating between the two luminosities is very difficult with the available spectra. However, the eclipse duration covers $\sim 0.25$ of the orbit (Hill et al. 2005). With a period of only $4.6 \mathrm{~d}$, a B1 Iab supergiant would not fit into the orbit. Therefore, a luminosity class Ib is definitely assigned to the donor. This locates the source at a distance of $d \approx 12.4 \mathrm{kpc}$, in agreement with Chaty et al. (2008), between the Sagittarius and Perseus arms, well beyond the Galactic center (Fig. 8).

Given this classification, the observed color excess is $E(B-$ $V) \approx 3$ and, assuming a standard extinction law, the extinction in the visual band would be $A_{V} \approx 9.4$. This is slightly higher than the value given by Chaty et al. (2008) $\left(A_{V} \approx 8.8\right)$ and by Masetti et al. (2008), namely $A_{V} \approx 8$.

For a standard reddening law (Fitzpatrick 1999), and typical intrinsic colors $(I-J)_{0}=-0.15,(R-I)_{0}=-0.17$ (Ducati et al. $2001)$, our value $E(J-K) \approx 1.54$ implies that $I \approx 14.8$ and $R \approx 16.9$ in excellent agreement with the value $R=16.9 \pm 0.1$ measured by Masetti et al. (2008).

\subsection{SAX J18186-1703}

SAX J1818.6-1703 was discovered by BeppoSAX during a strong outburst with a fast rise time of $\sim 1 \mathrm{~h}$, in March 1998 (in't Zand et al. 1998). Subsequently INTEGRAL detected a double-peaked outburst in September 2003 (Grebenev \& Sunyaev 2005) and two more in October 2003 (Sguera et al. 2005). Other fast outbursts were observed with the ASM onboard RossiXTE (Sguera et al. 2005). Its counterpart, 2MASS J18183790-1702479, was identified using a Chandra localization (in't Zand et al. 2006a). Using INTEGRAL, Zurita-Heras \& Chaty (2009), have reported a $22-50 \mathrm{keV}$ flux of the order of $(2-8) \times 10^{-11} \mathrm{erg} \mathrm{s}^{-1} \mathrm{~cm}^{-2}$ in quiescence, which reaches $(1-2) \times 10^{-9} \mathrm{erg} \mathrm{s}^{-1} \mathrm{~cm}^{-2}$ in the strongest flares. No

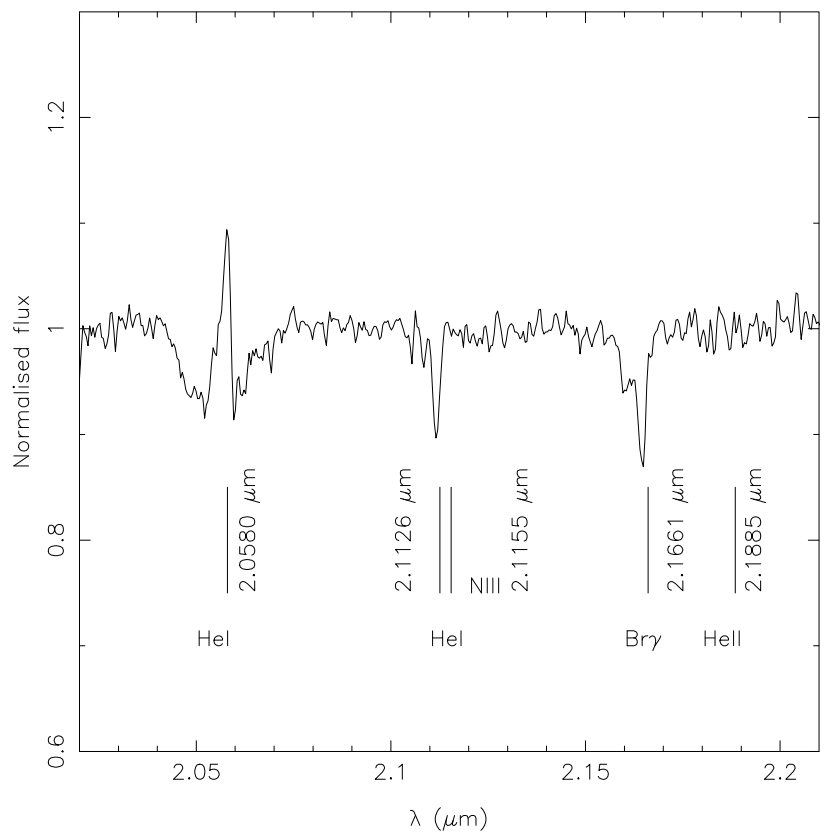

Fig. 2. $K$ band spectrum of the counterpart to SAX J18186-1703. Note the absence of any feature at the position of the He II $2.1885 \mu \mathrm{m}$ line.

pulsations have been detected so far, but an orbital period of $30 \mathrm{~d}$ has been established (Zurita-Heras \& Chaty 2009).

$K$ BAND SPECTRUM. In Fig. 2, we present the $K$ band spectrum of the IR counterpart. The absence of He II $2.1885 \mu \mathrm{m}$ confirms that this star is later than $\sim \mathrm{O} 8$. The He I $2.0581 \mu \mathrm{m}$ line is strongly in emission, as is typical of early B supergiants. Along with the morphology of the complex He I 2.1607/2.1617 - Br $\gamma$, appears to be of high luminosity. Main-sequence early B stars have broader lines (Hanson et al. 2005, Fig. 12). Following the same arguments presented for IGR J18027-2017, we assign a similar spectral type to this object and conclude that it is a supergiant in the B0-B1.5 range. The exact luminosity class is difficult to determine, but, as discussed previously, it is likely to be at least Iab. This would place the source at a distance of $d \approx 2.1 \mathrm{kpc}$ in the outskirts of the Sagittarius arm (Fig. 8).

\subsection{IGR J18483-0311}

IGR J18483-0311 is a transient X-ray source discovered by Chernyakova et al. (2003) during observations of the Galactic center with INTEGRAL. Using Swift, Sguera et al. (2007) suggested that a suitable counterpart is the IR source 2MASS J18481720-0310168, which is confirmed by Chaty et al. (2008). The X-ray source shows very high obscuration $\left(N_{\mathrm{H}}=9_{-4}^{+5} \times 10^{22} \mathrm{~cm}^{-2}\right)$. The system contains a pulsar $\left(P_{\mathrm{S}}=21.0526 \mathrm{~s}\right)$ in orbit around the primary with a period of $P_{\text {orb }}=18.52 \mathrm{~d}$ (Sguera et al. 2007). The unabsorbed X-ray 20-100 keV flux, measured with IBIS onboard INTEGRAL, is $\sim 2 \times 10^{-9} \mathrm{erg} \mathrm{s}^{-1} \mathrm{~cm}^{-2}$ (Sguera et al. 2007).

By comparing a low-resolution $H K$ spectrum to spectra of classification standards, Rahoui \& Chaty (2008) conclude that the counterpart is a B0.5 supergiant, and suggest a luminosity class Ia. In Fig. 3, we present the $I$ band spectrum of this object, between those of two supergiants of well-determined spectral type. Our spectrum claerly exhibits narow Paschen lines, typical of a high-luminosity object. Moreover, Paschen lines are visible up to $\mathrm{Pa} 18$, in spite of the moderate SNR and resolution, clearly identifying the star as a supergiant (compare to the 


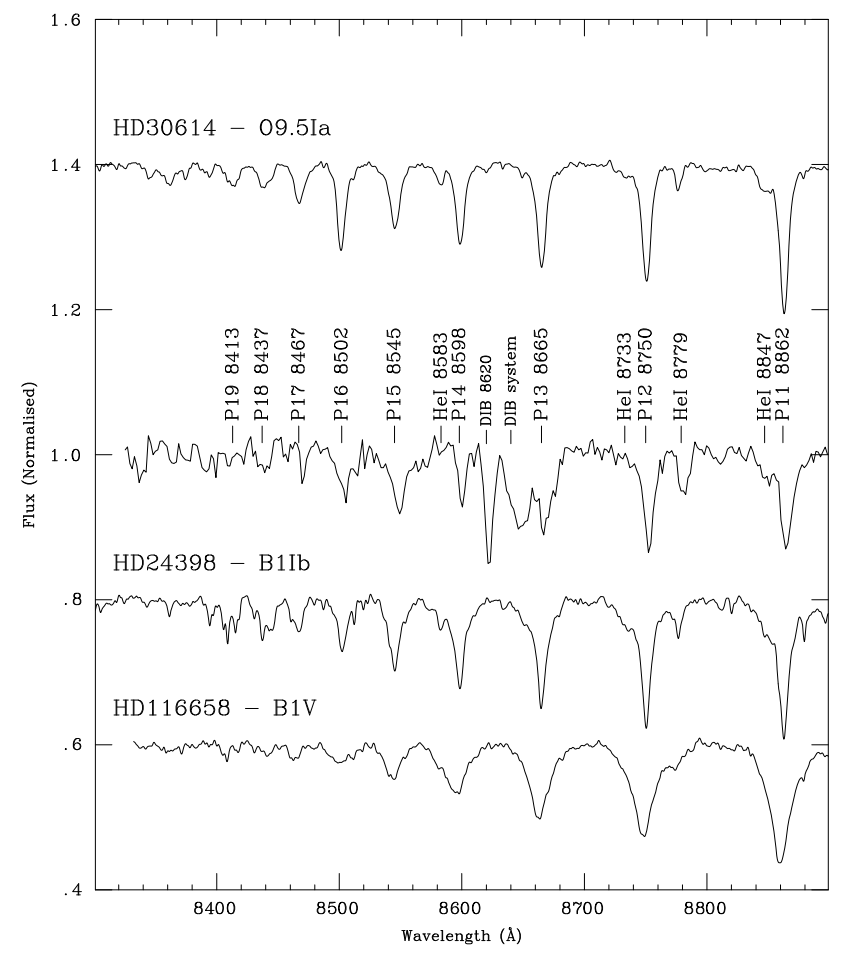

Fig. 3. WHT - I band spectrum of IGR J18483-0311.

B1 V star in the same figure). The presence of strong He I $8779 \AA$ is typical of early B supergiants, while the absence of O I $8446 \AA$ and $\mathrm{N}$ I lines makes it earlier than B2. Pa 16 is not prominently stronger than $\mathrm{Pa} 17$, suggesting that it does not contain an important contribution from C III $8502 \AA$. As discussed above, this suggests a spectral type of B0.5 or later. The exact determination of the luminosity class is not easy with the data available. However, we only detect Paschen lines up to P18 suggesting that the star is not a Ia supergiant. Combining our data with the $H K$ spectrum of Rahoui \& Chaty (2008), we conclude that the star is a B0.5-B1 supergiant, most likely with a luminosity class Iab. This would locate the system at $d \sim 2.8 \mathrm{kpc}$.

\subsection{IGR J1914.0+0951}

IGR J19140+0951 was discovered by Hannikainen et al. (2004) with INTEGRAL. It displays X-ray characteristics typical of a SGXB. in't Zand et al. (2006b) used Chandra to pinpoint the IR counterpart, which was found to be 2MASS J19140422+0952577. A period of $13.55 \mathrm{~d}$ is observed with RossiXTE (Corbet et al. 2004). No pulsations have been detected so far.

in't Zand et al. (2006b) presented a low resolution red spectrum of the source, which was very badly affected by fringing. It shows no counts bluewards of $7000 \AA$. They also quote a magnitude of $I=13.0$ for the counterpart, completely at odds with our value, and the low counts in the corresponding region of the spectrum.

Unfortunately, our I-band spectrum, although not affected by fringing is of low resolution and SNR. We identify a few narrow, strong Paschen lines, which are of indicative of an early-type supergiant. We do not detect the O I $7774 \AA$ line, which (given the low SNR in this part of the spectrum) constraints it to be earlier than $\sim \mathrm{B} 3$.
Hannikainen et al. (2007) presented a low SNR $K$-band spectrum and an $H$-band spectrum of much better resolution and SNR. Their analysis led them to conclude that the star was a B0.5 Ia supergiant. Nespoli et al. (2008) classify it as B1 Iab, based on a $K$-band spectrum. The spectrum presented by Hannikainen et al. (2007) displays Bry weakly in absorption, while this line is more clearly in absorption in the spectrum of Nespoli et al. (2008). However, our TNG spectra, taken four months later, as well as our SPEX spectra, taken one year earlier (both of higher SNR), show a P-Cygni profile in this line. This is an indication of high activity in this object and is consistent with the high luminosity of the star.

No He II absorption lines are seen in our spectra, suggesting that the star is later than O9. The presence of a very clearly defined series of Brackett $\mathrm{HI}$ lines in the $H$ band (see Fig. 4, $H$ band panel) also points towards an early B star, since these lines disappear for $\mathrm{O}$ type stars. The narrowness and depth of the $\mathrm{Br}$ lines is a clear indication of high luminosity. We also detect them well up to $\mathrm{Br} 19$, at least. The narrow and deep He I $1.7 \mu \mathrm{m}$ line is also typical of a supergiant. It is deeper than neighbouring $\mathrm{Br}$ lines, indicative of high luminosity. Its relative depth with respect to a nearby $\mathrm{Br} 11$ points clearly towards type B0-B0.5Ia and excludes types earlier than 09.7I (Hanson et al. 1998) (Fig. 2).

In addition, we find that He I $2.0581 \mu \mathrm{m}$ is strongly in emission, a characteristic of luminous early-B supergiants. This emission seems to be variable, because it is far more pronounced in the TNG data than in the IRTF observations (Fig. 4). We also detect emission in the $\mathrm{He} \mathrm{I} 1.083 \mu \mathrm{m}$ line in the $J$ band, and in $\mathrm{Pa} \beta$.

We conclude that the spectral type is B0.5 Ia, compatible with that given by Hannikainen et al. (2004) and Nespoli et al. (2008), but infer a slightly higher luminosity class. Nespoli et al. (2008) estimate a distance of $\approx 1.1 \mathrm{kpc}$, but they use the 2 MASS photometry, contaminated by the very bright star towards the upper right (see Fig. 5), namely 2MASS J19140417+0952538 $\left(K_{\mathrm{S}} \approx 6.5 \mathrm{mag}\right)$, as was already recognised by in't Zand et al. (2006b). We performed our photometry on images of higher spatial resolution that allowed us to clearly resolve both stars. We used an aperture small enough to ensure that the contribution from the bright star is minimal. We then applied an aperture correction to our magnitudes keeping in mind that avoiding the contamination completely is probably impossible without adaptive optics. Using our photometry, rather than the 2MASS values, and the inferred spectral type, we compute a distance of $d \approx 3.6 \mathrm{kpc}$.

In Fig. 6, we use our red and infrared photometry for this object to plot the RIJHK photometry absolute flux calibration, dereddened with a standard reddening law and assuming $E(B-V)=5.5$. The photometry is superimposed on a Kurucz atmosphere model for $T_{\text {eff }}=28000 \mathrm{~K}$ and $\log g=3.5$, corresponding to an early-type B supergiant ${ }^{3}$. The acceptable fit shows that the extinction to this source is close to standard, in spite of its high value. The normalization constant in Fig. 6, assuming $R_{*} \approx 30 R_{\odot}$, would yield a distance of $\approx 3.6 \mathrm{kpc}$, in agreement with the value computed previously. The independent estimate by Rahoui \& Chaty (2008), based on a fit to the near- and mid-infrared magnitudes, of $d \sim 3.1 \mathrm{kpc}$, is also consistent with our value.

\footnotetext{
3 Actually, $\log g=3.0$ would be more adequate. Unfortunately, this Kurucz model is not available. The difference in slope, however, is negligible for the present purposes.
} 
J. M. Torrejón et al.: Near-infrared survey of high mass X-ray binary candidates
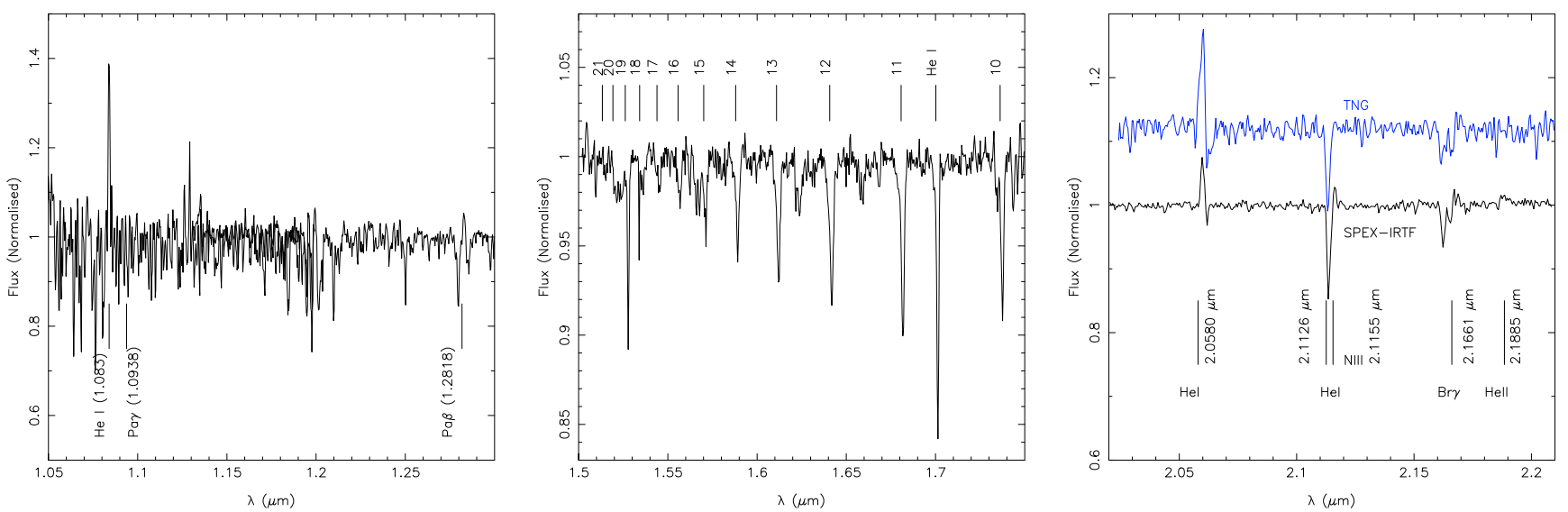

Fig. 4. SPEX-IRTF $J, H$ and $K$ band spectra of IGR J19140+0951. In the $K$ band, TNG data (top) are also presented.

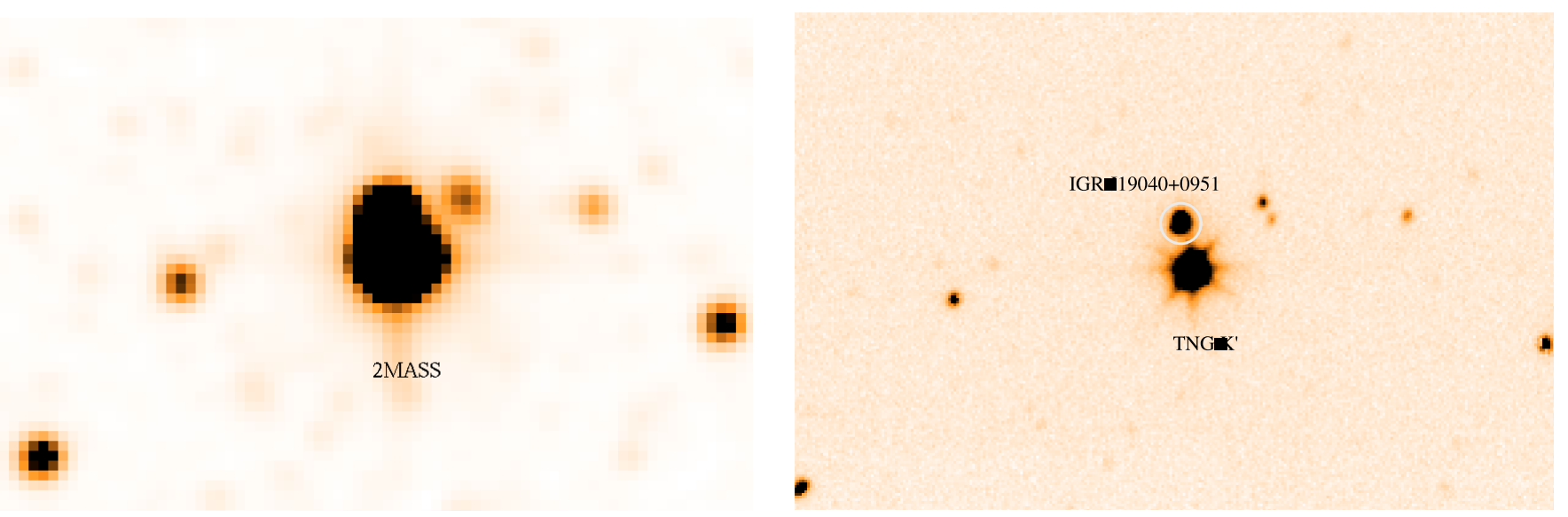

Fig. 5. The counterpart to IGR J19140+0951. Left panel: $K$-band image from 2MASS, showing the contamination by the light of the bright star 2MASS J19140417+0952538. Right panel: TNG $K^{\prime}$ band image, showing the two stars clearly resolved (cf. Fig. 2 in Rahoui \& Chaty (2008).)

\subsection{XTE J1901+014}

The source XTE J1901+014 was discovered by the RXTE/ASM monitor in 2002 during a brief outburst, after which the source went into quiescence below detectable limits. Examination of archival data showed that the source had undergone a previous brief outburst in 1997 (Remillard \& Smith 2002) showing a behaviour reminiscent of that of SFXTs. Smith et al. (2007) used $X M M$-Newton to obtain accurate positions of the X-ray source, which was found to be $\alpha=19 \mathrm{~h} 01 \mathrm{~m} 40.20 \mathrm{~s}, \delta=+01^{\circ} 26^{\prime} 26^{\prime \prime} .^{\prime} 0$ with an error of $\sim 1^{\prime \prime}$. We subsequently searched the area for possible counterparts. In Fig. 7, we show a $K^{\prime}$-band image of the area encompassing the error circle. Only one possible candidate is visible within the $X M M-N e w t o n$ error circle. The object is very faint with $K^{\prime} \gtrsim 14.2$, close to the limit of the sky brightness at $2 \mu \mathrm{m}$ at the La Palma Observatory. No sources show up in the $J$ or $H$-band images.

As the infrared observations do not provide enough information, we must resort to X-ray data in order to ascertain the nature of the system. The XMM-Newton spectra can be fitted well by a thermal bremsstrahlung with $k T \sim 6 \mathrm{keV}$ and an absorption column of $N_{\mathrm{H}} \sim 2.5 \times 10^{22} \mathrm{~cm}^{-2}$ (Rampy 2009, priv. comm.). This would correspond to $E(B-V) \approx 3.7$ (Ryter 1996), which is higher than the integrated value for the Milky Way galaxy in the direction of the source, namely $\sim 8.5 \times 10^{21} \mathrm{~cm}^{-2}$, suggesting intrinsic absorption, as might be expected. Assuming that all absorption is interstellar, the corresponding infrared excess is $E(J-K) \approx 1.8$. Comparing these values with those shown in

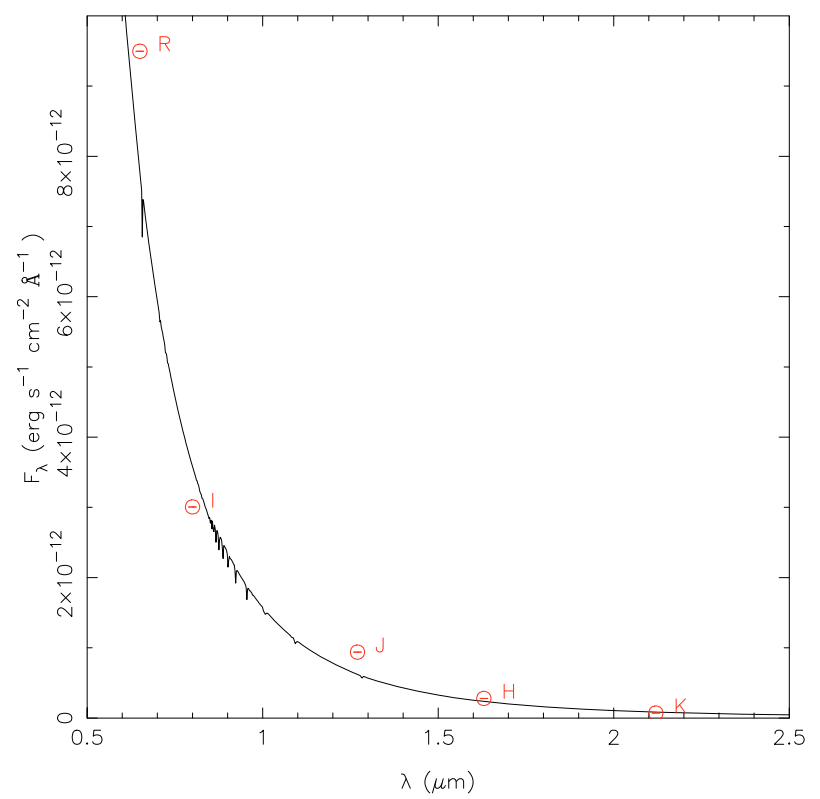

Fig. 6. Our RIJHK photometry of IGR J19140+0951 dereddened with a standard extinction law and $E(B-V)=5.5$ appears superimposed on a Kurucz atmosphere model for $T_{\text {eff }}=28000 \mathrm{~K}$ and $\log g=3.5$, corresponding to an early-type B supergiant.

Table 3, we can see that the source is, indeed, very reddened but not particularly so. 


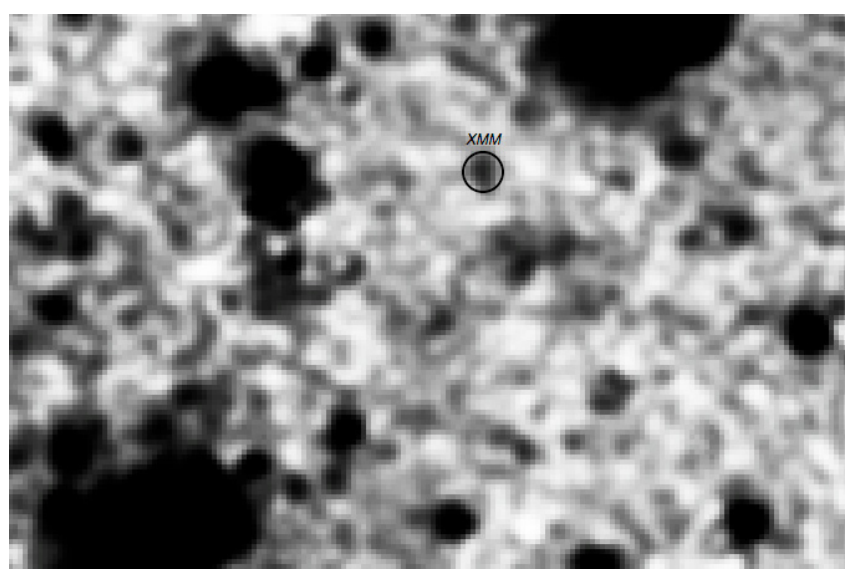

Fig. 7. TNG $K^{\prime}$-band image of the field around XTE J1901+014 and the nominal XXM-Newton position with an error radius of $1^{\prime \prime}$ (which for our image scale is 4 pixels). It is not visible in either the $J$ or $H$ bands.

If we assume an $M_{K}$ typical of a not very luminous OB supergiant $(\sim-5.5$; Martins \& Plez 2006$)$ with $K \approx 14.2$, the distance to the source would be $\sim 47 \mathrm{kpc}$, placing it well outside the Galaxy. This seems very unlikely. At the outskirts of the Galaxy in the direction to the source $(\sim 22 \mathrm{kpc})$, the infrared magnitude of the counterpart would be $M_{K} \approx-3.8$. This would correspond to a $\mathrm{O} 8 \mathrm{~V}$ star or a K5 III star. Therefore the possibility of a supergiant companion is excluded.

However, there are arguments to believe that the distance must be far lower. Indeed, the 2002 outburst detected with RossiXTE/ASM reached a luminosity of the order of 1 Crab, which corresponds to a flux $F_{\mathrm{X}}=3.63 \times 10^{-8} \mathrm{erg} \mathrm{cm}^{-2} \mathrm{~s}^{-1}$. At a distance $d=22 \mathrm{kpc}$, the outburst should have reached a $L_{\mathrm{X}} \approx 2 \times 10^{39} \mathrm{erg} \mathrm{s}^{-1}$. This is equivalent to the Eddington luminosity of a compact object of $\sim 16 M_{\odot}$, and higher than ever reported for any neutron star system. On the other hand, if we assume that the outburst luminosity was the Eddington luminosity for accretion onto a NS $\left(\sim 2 \times 10^{38} \mathrm{erg} \mathrm{s}^{-1}\right)$, the maximum distance to the source should be of the order of $d_{\max } \sim 7 \mathrm{kpc}$, which would place the source at the tip of the Long Bar in the Galactic nucleus. For such a source, $M_{K} \approx-1.28$, which would be compatible with a G5 III star. The maximum distance at which the primary would be compatible with a class III star is $d \sim 5 \mathrm{kpc}$, in which case, we would infer a class of G4III (or also B4V). Below this distance, essentially, only main-sequence stars are allowed. For distances around $1 \mathrm{kpc}$ or less, the only compatible companions would be $\mathrm{G}, \mathrm{K}$, or $\mathrm{M}$ main-sequence stars. This raises the interesting possibility that the system is a cataclysmic variable, since the X-ray spectrum can be described by a thermal bremsstrahlung at $6 \mathrm{keV}$. However, in such a case, $L_{\mathrm{X}} \sim 10^{36} \mathrm{erg} \mathrm{s}^{-1}$, which would be several orders of magnitude higher than usually found for CV. Therefore, we conclude that, very likely, $d_{\min } \sim 1-2 \mathrm{kpc}$. This is in good agreement with the conclusions obtained by Karasev et al. (2008a,b), who argue in favor of a distance of $\sim 5 \mathrm{kpc}$.

\section{Discussion and conclusions}

Four of our sources have early-B supergiant companions. All four counterparts are found to have spectra that cover a rather narrow spectral interval, being early-B (B0-B1.5) supergiants of moderate or high luminosity. This identifies the nature of the X-ray source unambiguously as HMXBs. Furthermore, they are all heavily obscured, with $E(B-V) \sim 3-5.5$, implying extinctions on the order of $A_{V}=9-16$ mag in the visual band. On the other hand, XTE 1901+014 has no obvious counterpart in any band except $K^{\prime}$. Thus, a supergiant nature is definitely excluded, thereby setting it apart from the other four.

IGR J18027-2017 is a persistent X-ray source with a counterpart around B1 Ib. It is therefore, a SGXB system. The reason why it has not yet been detected by earlier X-ray missions is unclear. For example, it was not detected by ROSAT. At a distance of $d \approx 12.4 \mathrm{kpc}$, its XMM-Newton detected flux in the $2-10 \mathrm{keV}$ band for the main pulse of $8.9 \times 10^{-11} \mathrm{erg} \mathrm{s}^{-1} \mathrm{~cm}^{-2}$, would yield a luminosity of $L_{\mathrm{X}}=1.65 \times 10^{36} \mathrm{erg} \mathrm{s}^{-1}$. This is typical of these systems albeit among the lower values. Since the detected flux outside the primary pulse is a little bit lower, the system can spend a fraction of its time in the upper $10^{35} \mathrm{erg} \mathrm{s}^{-1}$. Given its high obscuration, this can explain why it was missed by earlier surveys.

For SAX J18186-1703, the NIR counterpart is a B0.5Iab star, confirming its nature as a SFXT. The $22-50 \mathrm{keV}$ flux reported by Zurita-Heras \& Chaty (2009) is of the order of $(2-8) \times 10^{-11} \mathrm{erg} \mathrm{s}^{-1} \mathrm{~cm}^{-2}$ in quiescence, while it reaches $(1-2) \times$ $10^{-9} \mathrm{erg} \mathrm{s}^{-1} \mathrm{~cm}^{-2}$ for the strongest flares. At the inferred distance of $\sim 2.1 \mathrm{kpc}$, the X-ray luminosity of this object would be $\sim 3 \times 10^{34} \mathrm{erg} \mathrm{s}^{-1}$ in quiescence, while the peak luminosity can be as high as $\sim 8 \times 10^{35} \mathrm{erg} \mathrm{s}^{-1}$. This is lower than those found in other SFXTs $\left(\sim 10^{36} \mathrm{erg} \mathrm{s}^{-1}\right)$. Zurita-Heras \& Chaty (2009) found that this system is in a very eccentric orbit $(e \sim 0.3-0.4)$, with $P_{\text {orb }} \approx 30 \mathrm{~d}$, amongst the largest yet found for SFXT, reaching a periastron distance between 2 and $3 R_{*}$. This would locate the compact object at a slightly larger distance from the donor than in the SGXB systems $\left(a \lesssim 2 R_{*}\right)$ thereby reducing the $L_{X}$ of the outbursts slightly below $10^{36} \mathrm{erg} \mathrm{s}^{-1}$, as observed.

IGR J18483-0311 is a transient system with a B0-B1 Iab primary. It has been classified as an intermediate SFXT (Rahoui \& Chaty 2008). Like many other SFXTs, it is a nearby source at $\sim 2.8 \mathrm{kpc}$. For this distance, the INTEGRAL/IBIS $20-100 \mathrm{keV}$ flux (Sguera et al. 2007) translates into a luminosity $\sim 1.9 \times$ $10^{36} \mathrm{erg} \mathrm{s}^{-1}$, for the strongest flares, which is typical of this kind of object. The orbital period of this system is $18.5 \mathrm{~d}$, while the spectral type of the companion is similar to that of SAX J18186-1703. Therefore, the compact object would be located slightly closer to the star and, correspondingly, its average $\mathrm{X}$-ray luminosity will be higher, in agreement with the scenario described in Negueruela et al. (2008).

IGR J19140+0951 is a persistent X-ray source and therefore, owing to its counterpart, a SGXB located at $d \approx 3-4 \mathrm{kpc}$, a little bit further than previously thought. For the bright INTEGRAL flux in the $2-20 \mathrm{keV}$ band of $1 \times 10^{-9} \mathrm{erg} \mathrm{s}^{-1} \mathrm{~cm}^{-2}$ (Hannikainen et al. 2004), the $L_{X}=1-2 \times 10^{36} \mathrm{erg} \mathrm{s}^{-1}$, typical of a SGXB, albeit, again, at the fainter end. Together with IGR 18027-2017, they have lower X-ray fluxes than the average SGXB, which explains their more recent detections in the newer, and deeper INTEGRAL observations.

As can be seen in Fig. 8, the sources tend to concentrate in or behind the section of the Sagittarius arm projected onto the direction of the Galactic bulge, an area intensively scanned by INTEGRAL. They are, thus, relatively close to the Sun (in comparison to typical distances to HMXBs), although highly reddened. This can introduce a bias in the newly detected populations of obscured sources, which tend to harbor SG companions, since only the brighter and/or closer ones can be reached with telescopes of moderate apertures in the $4 \mathrm{~m}$ class. A transient system with a main-sequence companion, such as a BeXB, will be too faint to be conclusively classified (i.e.; Zurita-Heras $\&$ Chaty 2008). Remarkably, the furthest source in our sample is 


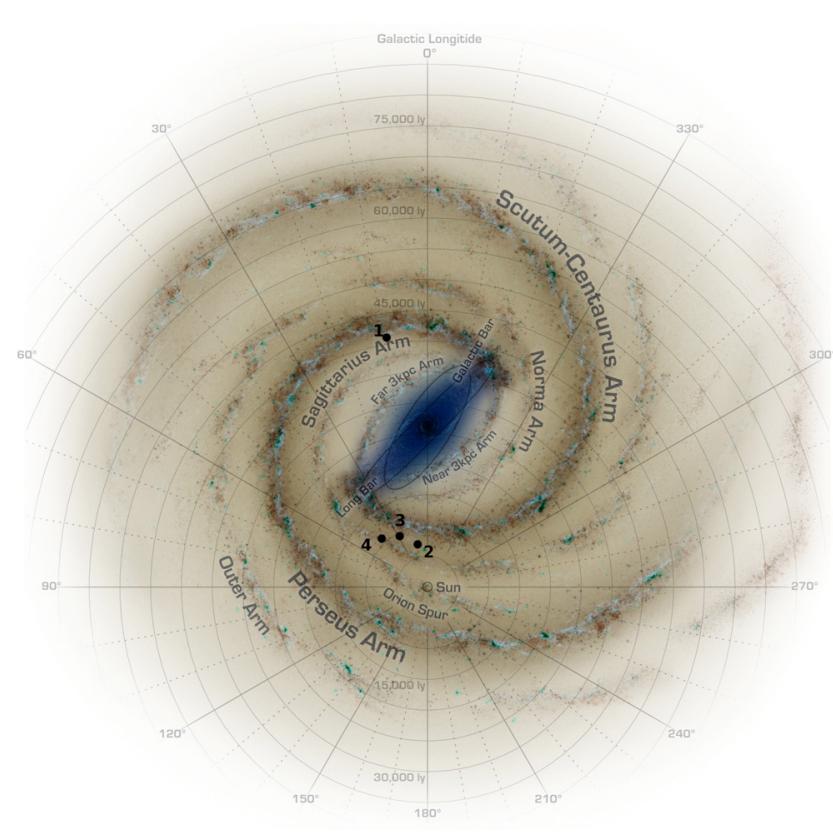

Fig. 8. Sketch of the Galaxy showing the position of the HMXBs studied in this work (filled dots). Numbers refer to the relative order of the source in Table 3. Galaxy model from Churchwell et al. (2009).

the least reddened one. This raises the question of the true abundance of HMXBs (and OB stars in fact) in our Galaxy. As can be seen in Table 3, the high reddening is not a sufficient condition to be an SFXT, since some SGXBs (such as IGR J19140+0951) are more reddened. On the other hand, it is not a necessary condition either, as other SFXTs exhibit rather low reddenings (for example, IGR J08408-4503 with $E(B-V)=0.5$ Negueruela et al. 2009). Furthermore, the position in the arms of the Galaxy of the newly discovered HMXBs (including the SFXTs) is entirely consistent with the positions of the previously known HMXBs (including SGXBs). Therefore, there is no reason to believe that they form a different population. This would be expected from a scenario similar to that discussed in Negueruela et al. (2008). In this scenario, the SFXTs are proposed to be SGXBs in which the compact object is located further out in the system, in a region where the stellar wind of the supergiant companion has become substantially clumped. The number of HMXBs in the Galaxy would then be much higher than previously thought. But so would the number of isolated OB stars, whose much lower $\mathrm{X}$-ray emission will not be detected in current X-ray surveys if they are heavily obscured. This is supported by the discovery of a growing population of very massive infrared clusters (e.g., Crowther et al. 2006; Messineo et al. 2008). Therefore, in principle, there is no reason to believe that the relative abundance of HMXBs, with respect to OB stars, differs from the current values, which are well explained by theoretical models.

Finally, XTE J1901+014 emerges as a transient source whose X-ray behavior is characteristic of SFXTs but where the presence of a supergiant companion is clearly ruled out. It may still be a massive star close to the main sequence in the outskirts of the Galaxy, perhaps with a $16 M_{\odot} \mathrm{BH}$ as a companion. In this respect, it is interesting to note the case of the LMC transient A $0535-66$ reaching $L_{\mathrm{X}} \approx 8 \times 10^{38} \mathrm{erg} \mathrm{s}^{-1}$ (e.g., Pakull \& Parmar 1981). A similar source at the edge of the Galaxy could have a similar hard X-ray behaviour. However, taking into account the moderate hardness (the source is observed as 1 RXH J190140.1+012630) and obscuration of the source, it seems more likely that the counterpart is a late-type star located between $2 \mathrm{kpc}$ and the Long Galactic bar $(7 \mathrm{kpc})$, somewhere between luminosity class V to class III, but definitely not consistent with a class I star. Whether a main sequence or giant late-type star wind can produce the instabilities necessary to produce the observed outbursts via accretion onto a compact object remains an open issue.

Acknowledgements. This research has been funded by grants AYA2008-06166C03-03 and Consolider-GTC CSD-2006-00070 from the Spanish Ministerio de Ciencia e Innovación (MICINN). JMT also acknowledges the research grant PR2007-0176. The authors would like to thank the referee, Dr. Alex Kaper, for his useful comments and suggestions.

The Nordic Optical Telescope is operated on the island of La Palma jointly by Denmark, Finland, Iceland, Norway, and Sweden, in the Spanish Observatorio del Roque de los Muchachos of the Instituto de Astrofisica de Canarias. The data were taken with ALFOSC, which is owned by the Instituto de Astrofísica de Andalucía (IAA) and operated at the Nordic Optical Telescope under agreement between IAA and the NBIfAFG of the Astronomical Observatory of Copenhagen.

The WHT is operated on the island of La Palma by the Isaac Newton Group in the Spanish Observatorio del Roque de Los Muchachos of the Instituto de Astrofísica de Canarias. The WHT data presented here have been obtained as part of the service programme. We thank the support astronomers for their dedication during the service nights.

Partly based on observations made with the Italian Telescopio Nazionale Galileo (TNG) operated on the island of La Palma by the Fundación Galileo Galilei of the INAF (Istituto Nazionale di Astrofisica) at the Spanish Observatorio del Roque de los Muchachos of the Instituto de Astrofisica de Canarias. This research has made use of the Simbad data base, operated at CDS, Strasbourg (France) and of data products from the Two Micron All Sky Survey, which is a joint project of the University of Massachusetts and the Infrared Processing and Analysis Center/California Institute of Technology, funded by the National Aeronautics and Space Administration and the National Science Foundation.

\section{References}

Andrillat, Y., Jaschek, C., \& Jaschek, M. 1995, A\&AS, 112, 475 Augello, G., Iaria, R., Robba, N. R., et al. 2003, ApJ, 596, 63 Bird, A. J., Malizia, A., Bazzano, A., et al. 2007, ApJ, 170, 175 Blum, R. D., Raymond, T. M., Conti, P. S., Figer, D. F., \& Sellgren, K. 1997, AJ, 113,1855

Chaty, S., Rahoui, F., Foellmi, C., et al. 2008, A\&A, 484, 783 Churchwell, E., Babler, B. L., Meade, M. R., et al. 2009, PASP, 121, 213 Chernyakova, M., Lutovinov, A., \& Capitanio, F. 2003, ATel, 157 Clark, J. S., Steele, I. A., Fender, R. P., \& Coe, M. J. 1999, A\&A, 348, 888 Corbet, R. H. D., Hannikainen, D. C., \& Remillard, R. 2004, ATel, 269

Crowther, P. A., Hadfield, L. J., Clark, J. S., Negueruela, I., \& Vacca, W. D. 2006, MNRAS, 372, 1407

Ducati, J. R., Bevilacqua, C. M., Rembold, S. B., \& Ribeiro, D. 2001, ApJ, 558, 309

Fitzpatrick, E. L. 1999, PASP, 111, 63

Grebenev, S. A., \& Sunyaev, R. A. 2005, Ast. Lett., 31, 672

Halpern, J. P., \& Gotthelf, E. V. 2004, ATel, 344

Halpern, J. P., \& Gotthelf, E. V. 2007, ApJ, 669, 579

Hannikainen, D. C., Rodriguez, J., Vilhu, O., et al. 2005, A\&A, 435, 995

Hannikainen, D. C., Rawlings, M. G., Muhli, P., et al. 2007, MNRAS, 380, 665

Hanson, M. M., Conti, P. S., \& Rieke, M. J. 1996, ApJS, 107, 281

Hanson, M. M., Rieke, G. H., \& Luhman, K. L. 1998, AJ, 116, 191

Hanson, M. M., Kudritzki, R. P., Kenworthy, M. A., Puls, J., \& Tokunaga, A. T. 2005, ApJS, 161, 154

Harrison, T. E., Howell, S. B., Szkody, P., et al. 2004, ApJ, 614, 947

Hill, A. B., Walter, R., Knigge, C., et al. 2005, A\&A, 439, 255

Hunt, L. K., Mannucci, F., Testi, L., et al. AJ, 115, 2594

in't Zand, J., Heise, J., Smith, M., et al. 1998, IAUC, 6840, 2

in't Zand, J. J. M., Jonker, P., Mendez, M., \& Markwardt, C. 2006a, ATel, 915

in't Zand, J. J. M., Jonker, P. G., Nelemans, G., Steeghs, D., \& O'Brien, K. 2006b, A\&A, 448, 1101

Karasev, D. I., Lutovinov, A. A., \& Burenin, R. A. 2008a, Astron. Lett., 34, 753

Karasev, D. I., Lutovinov, A. A., \& Burenin, R. A. 2008b, Pisma v Astron.Zh., 34 (2008), 834

Kleinmann, S. G., \& Hall, D. N. B. 1986, ApJS, 62, 501

Maiolino, R., Rieke, G. H., \& Rieke, M. J. 1996, AJ, 111, 537

Malizia, A., Bassani, L., Stephen, J. B., et al. 2005, ApJ, 630, 157

Martins, F., \& Plez, B. 2006, A\&A, 457, 637

Masetti, N., Mason, E., Morelli, L., et al. 2008, A\&A, 482, 113 
Mason, A. B., Clark, J. S., Norton, A. J., Negueruela, I., \& Roche, P. 2009, A\&A, 505,281

Messineo, M., Figer, D., Davies, B., et al. 2008, ApJ, 683, 155

Meyer, M. R., Edwards, S., Hinkle, K. H., \& Strom, S. E. 1998, ApJ, 508, 397

Negueruela, I., Smith, D. M., Reig, P., et al. 2006a, ESA SP-604, 165

Negueruela, I., Smith, D. M., Harrison, T. E., \& Torrejón, J. M. 2006b, ApJ, 638, 982

Negueruela, I., Torrejón, J. M., Reig, P., et al. 2008, AIPC, 1010, 252

Negueruela, I., Torrejón, J. M., \& Reig P. 2009, in Proceedings of the 7th INTEGRAL Workshop - An INTEGRAL View of Compact Objects, Copenhagen, Denmark, 8-11 September 2008, Proc. Science

Nespoli, E., Fabregat, J., \& Mennickent, R. E. 2008, A\&A, 486, 911

Pakull, P., \& Parmar, A. 1981, A\&A, 102, L1

Rahoui, F., \& Chaty, S. 2008, A\&A, 492, 163

Rahoui, F., Chaty, S., Lagage, P.-O., \& Pantin, E. 2008, A\&A, 484, 801

Remillard, R., \& Smith, D. 2002, ATel, 88
Revnivtsev, M. G., Sunyaev, R. A., Varshalovich, D. A., et al. 2004, Astron. Lett., 30,382

Ryter, Ch. E. 1996, Ap\&SS, 236, 285

Schmidt-Kaler, T. H. 1982, in Stars and Star Clusters, Landolt-Bornstein New Series, Vol. 2b, Astronomyand Astrophysics - ed. K. Schaifers, \& H. H. Voigt (New York: Springer-Verlag),

Sguera, V., Barlow, E. J., Bird, A. J., et al. 2005, A\&A, 444, 221

Sguera, V., Bazzano, A., Bird, A. J., et al. 2006, ApJ, 646, 452

Sguera, V., Hill, A. B., Bird, A. J., et al. 2007, A\&A, 467, 249

Smith, D. M., Heindl, W. A., Markwardt, C. A., et al. 2006, ApJ, 638, 974

Smith, D. A., Rampy, R. A., Negueruela, I., \& Torrejón, J. M. 2007, ATel, 1268

Wallace, Ll., Meyer, M. R., Hinkle, K., \& Edwards, S. 2000, ApJ, 535, 325

Walter, R., Zurita-Heras, J., Bassani, L., et al. 2006, A\&A, 453, 133

Wegner, W. 1994, MNRAS, 270, 229

Wegner, W. 2006, MNRAS, 371, 185

Zurita-Heras, J., \& Chaty, S. 2008, A\&A, 489, 657

Zurita-Heras, J., \& Chaty, S. 2009, A\&A, 493, L1 